\title{
RESEARCHERS AT THE JACKSON HOLE RESEARCH STATION AND THEIR INSTITUTIONAL AFFIIIATIONS \\ 1946-1985
}

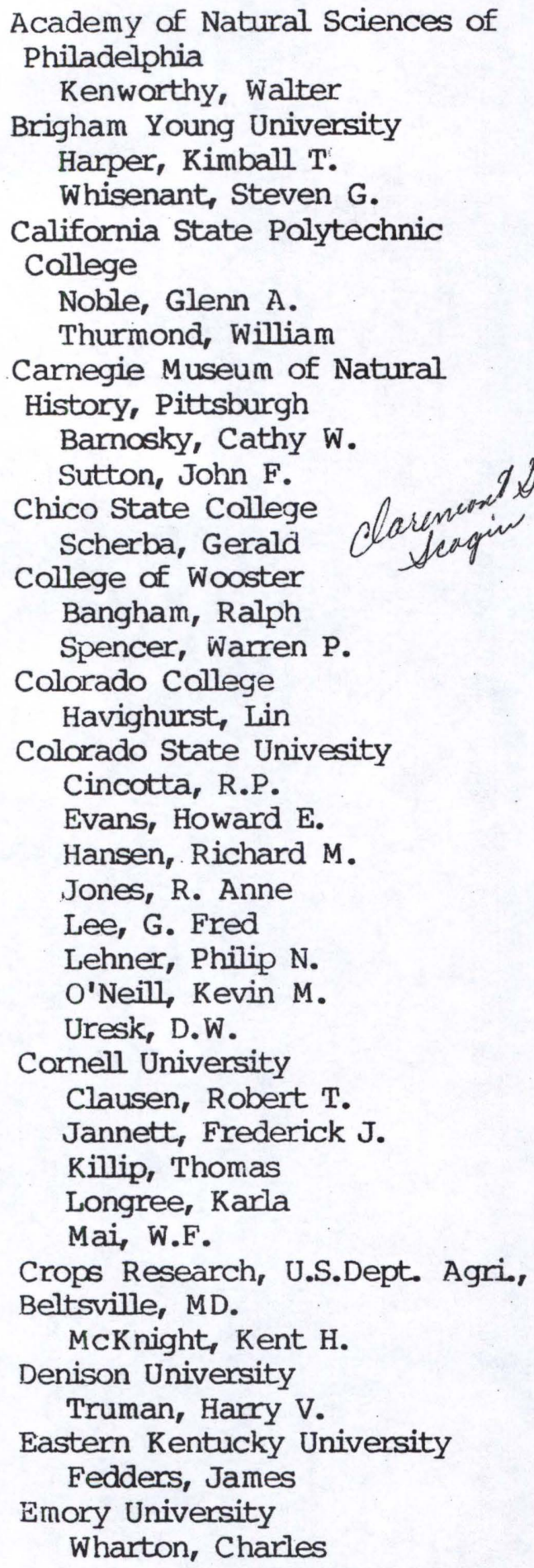

Flint Junior College Merkle, John

Fort Lewis College Romme, William $\mathrm{H}$.

Franklin and Marshall College John, Kenneth R.

Gettysburg College Ruos, James

Harvard University Evans, Howard E. Held, Richard Schneiderman, Howard A. ho State University Clark, Tim W. Keller, Barry L. Whitfield, Michael B.

Iowa State University Dahlgren, Robert $\mathrm{B}$. Garrett, Monte Franklin, William Popp, Jewel $\mathrm{K}$. wydeven, Adrian P.

Johns Hopkins University Sladen, william J.

Kenyon College Anderson, Stanley $\mathrm{H}$. Thornton, Charles

Lawrence College Kennington, Garth

Loma Linda University Buchheim, H. Paul

Los Angeles State College Lowrie, Donald C.

Michigan Technological University Bartelli, Lindo J. Forde, Jon D. Shown, D.A. Sloan, Norman F. Trettin, C.C.

Montana State University Eng, Robert L. Eversman, Sharon Gould, William R. Irby, Lynn Marlow, Clayton B. 
McFeters, Gordon A.

Moore, R.E.

Norland, Jack E.

Oswald, Edward

Rumely, John H.

Vyse, Ernest R.

Weaver, Tad

Wood, M.A.

Woods, Bryant

Worley, David E.

Youmans, Clifton C.

Mount Holyoke

Kelsey, Elizabeth B.

Larson, Betty J.

North Dakota State University

Clambey, Gary K.

Northern Arizona University States, Jack S.

Oberlin College

Gregg, Peter A.

Peterson, James A.

Oregon State University

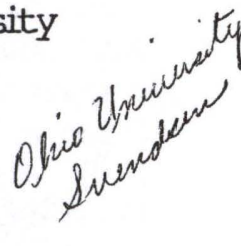
Jackson, Royal

Penn State College

Carpenter, C.R.

Collins, Thomas C.

Smalley, Alfred E:

Peregrine Fund Incorporated

Burnham, William

Purdue University

Cummins, George B.

Kuns, Merle L.

Rancho Santa Ana Botanic

Garden

Scogin, Ron L.

Rocky Mountain Forest and

Range Experiment Station,

Rapid City

Uresk, Dan W.

Saint Louis University

Feir, Dorothy

McClain, Elizabeth

Mulligan, C.R.

Mulligan, James A.

Watt, Gerald

San Francisco State University Hall, Joseph G.

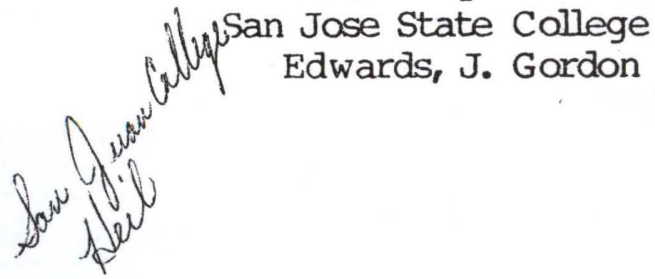

St. Olaf's College

Peterson, Arnold

Science Museum of Minnesota

Jannett, Frederick J., Jr.

Snow College

Blauer, A. Clyde

South Dakota State University

Gartner, F. Robert

White, Everett M.

Worcester, L.L.

State University of New York, Albany

Bender, Susan J.

Mackiewicz, John S.

Marceau, Thomas E.

Wright, Gary A.

Swarthmore College

Enders, Robert $\mathrm{K}$.

Meinkoth, Norman A.

Lettlier, Joe L.

Syracuse University Jannett, Janice

Texas Tech University Sutton, John F.

Tulane University

Gould, Edwin

Negus, Norman C.

University of Arizona

- Bagnara, Joseph T.

University of California, Davis

Gall, G.A.E.

Loudenslager, Eric J.

Salt, George $W$.

University of California, Los Angeles Cody, Martin L. Hutto, Richard L.

University of Chicago Foreman, Darhl L.

University of Colorado Altmann, Margaret Bock, Carl E. Bock, Jane $\mathrm{H}$.

Garst, Warren E.

Klein, Stephen

Medine, Allen

Stoecker, Robert E.

Williams, Olwen

University of Helsiniki

Terkivuo, Juhani 
University of mllinois MacLeod, Ellis G. Opsahl, James Tiver, Jack D. University of Kansas Armitage, Kenneth El-Buns, Abdalaziz M. Hall, E. Raymond Lichtwardt, Robert W. Roofe, Paul G.

University of

Massachusetts

Williams, Jeanne $\mathrm{H}$. University of Michigan Carpenter, Charles $C$. Craighead, F.C. Craighead, J.J. Gilligan, Jim McKnight, Karl B. Patterson, Robert L. Plaxton, Meredith J. Russell, Cynthia University of Minnesota Alexander, E. Calvin, Jr. Bennett, Donald V. Wright University of Montana Allen, Harriet L. Bromenshenk, Jerry J. Boldibact Crenshaw, J. Lowe, J.H. McClelland, B. Riley McClelland, P.T. Metzgar, Lee $\mathrm{H}$. Pfister, Robert D. Rice, Peter M. Shea, David S. Shea, Ruth E. Spettigue, Elizabeth B. Tourangeau, Phillip C. Weist, K.M. Willard, E.E. Wilson, P.B. Young, Leonard S. University of Nebraska Johnsgard, Paul A. Laing, Charles O'Dell, William D. Ramaley, Robert F. University of New Hampshire Milne, Lonus J.
Milne, Margery

University of New Mexico Gwynne, Darryl T.

University of New Orleans Englers, K. Dale Pinter, Aelita J. University of Northern Colorado Olmstead, Charles E.

University of Notre Dame Bick, George $\mathrm{H}$.

University of Reading, England Moss, Stephen T.

University of Rochester Maynard Elliott A.

University of Southern California Burbank, Douglas W. Nakamura, Mitsuru

University of South Dakota Hansen, Paul L. Hoffman, George R. Hopkins, Ricky

University of Texas Steele, James $\mathrm{H}$. University of Toronto Morris, Glenn K. University of Utah Allis, Richard G. Bow man, John R. Chapman, David S. Doser, Diane I. French, Norman Parry, William T. Smith, Robert B.

University of Washington Barnosky, Cathy W. Barnosky, Anthony D.

University of Wisconsin Emlen, John T. Flack, Douglas McHugh, Tom C. Petersen, Arnold Rausch, Robert Schaller, George B.

University of Wisconsin, Milwaukee Baxter, John Edgington, David N. Kaster, Jerry L. Klump, J. Val Piehl, Martin A. 


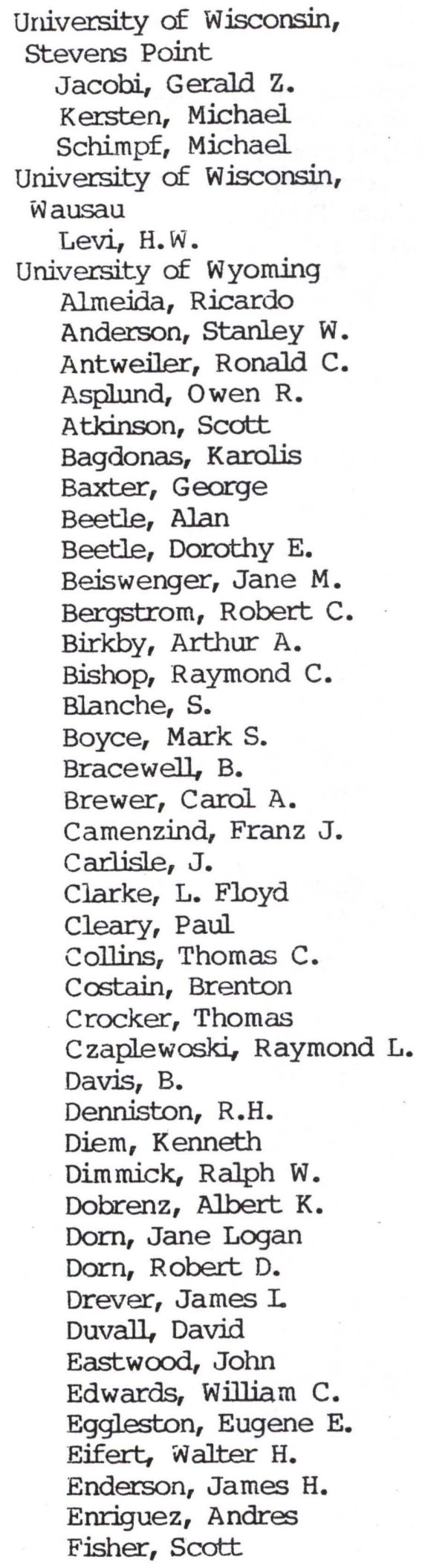

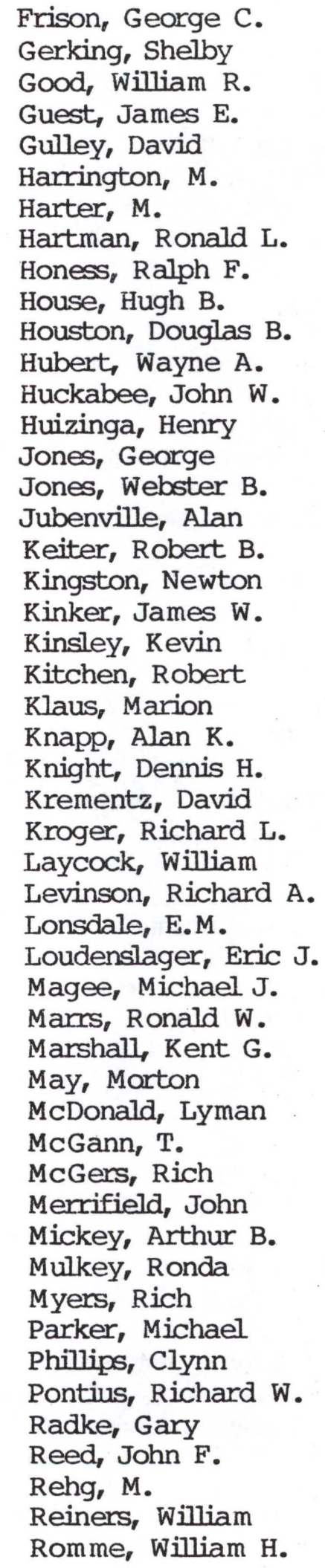

Frison, George C. Gerking, Shelby Good, William R. Guest, James E. Gulley, David Harrington, M. Harter, $M$. Honess, Ralph F. House, Hugh B. Houston, Douglas B. Hubert, Wayne A. Huckabee, John W. Huizinga, Henry Jones, George Jones, Webster B. Jubenville, Alan Keiter, Robert B. Kingston, Newton Kinker, James $\mathrm{W}$. insley, Kevin Klaus, Marion Knapp, Alan K. Krementz, David Kroger, Richard L. Laycock, William Levinson, Richard A. Lonsdale, E.M. Loudenslager, Eric J. Magee, Michael J. Marrs, Ronald $\mathrm{W}$. Marshall, Kent G. May, Morton McDonald, Lyman McGann, T. Merrifield, John Mickey, Arthur B. Mulkey, Ronda Myers, Rich Parker, Michael Phillips, Clynn Pontius, Richard W. Radke, Gary Reed, John F. Reiners, William Romme, William $\mathrm{H}$. 
Sabinske, Darold

Sauer, John R.

Skryja, David

Smith, Dixie R.

Smith, William $\mathrm{K}$.

Snow, Carol J.

Solheim, William G.

Speas, Janice

Stanton, Nancy

Steidtmann, James R.

Stoops, Paul D.

Swanson, Richard D.

Swatek, John A.

Taylor, Dale L.

Thompson, James G.

Thompson, Thomas

VanDusen, Larry

Waitman, Jerry

Warder, Donald S.

Wesche, Thomas A.

Wiley, Robert $\mathrm{W}$.

Wills, $B$.

Wilson, Eugene

Yavitt, J.B.

Zeveloff, Samuel I.

U.S. Army Institute of Pathology,

Washington, DC

Jones, T.C.

U.S. Forest Service

McArthur, E. Durant

Monsen, Stephen B.

U.S. Water and Power Resources

Service Engineering and

Research Center

Meeder, Chuck

Utah State University

Bassett, Neil R.

Fisher, Richard

Jenkins, Michael

Parker, Frank D.

Rudersdorf, Ward

Salesbury, Frank B.

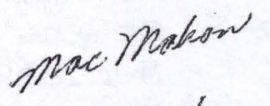

Tepedino, Vince $\mathrm{J}$.

Virginia Polytechnic Institute

Calder, William A.

Washington State University

Jonas, Robert J.

Pfister, Allan R.

Wayne State University

Jones, Lily A.

\author{
Wellesley College \\ Scott, Ann \\ Western Reserve University \\ Findley, James S. \\ Smith, Robert S. \\ Wyoming Game and Fish Department \\ Mitchum, Douglas \\ Wyoming Natural Heritage Office \\ Collins, Ellen
}

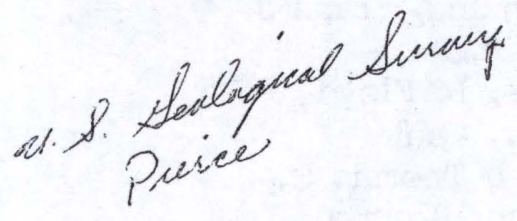

